\title{
DEL MISTERIO DE LA TRASCENDENCIA A LA MARAVILLA DE LA DONACIÓN: EL ENIGMA EN $L A$ IDEA DE $L A$ FENOMENOLOGÍA DE HUSSERL
}

\author{
Hernán G. Inverso* \\ doi: 10.11144/Javeriana.uph35-71.tdeh
}

\section{RESUMEN}

La idea de la fenomenología constituye el resultado del primer curso que Husserl diseñó para dar cuenta de las novedades disciplinares que trajo aparejado el giro trascendental ocurrido alrededor de 1905 . Se trata, por tanto, no solo de un documento importante de esta inflexión, sino también de una obra de alto impacto en la tradición fenomenológica posterior. En este trabajo nos detendremos en tres aspectos de la categoría de enigma (Rätsel): la manera como esta caracteriza a la trascendencia, la apelación a la figura de objetores y la cuestión de la donación y sus modos. Este recorrido permitirá poner de relieve aspectos centrales y poco explorados de la fenomenología trascendental en sus inicios.

Palabras clave: fenomenología; Husserl; método; trascendencia; donación

Universidad de Buenos Aires / ANCBA-CONICET.

Correo electrónico: hernaninverso@filo.uba.ar

Para citar este artículo: Inverso, H. G. (2018). Del misterio de la trascendencia a la maravilla de la donación: el enigma en La idea de la fenomenología de Husserl. Universitas Philosophica, 35(71), pp. 233-258. ISSN 0120-5323, ISSN en línea 2346-2426. doi: 10.11144/Javeriana.uph35-71.tdeh 


\title{
FROM THE MYSTERY OF TRANSCENDENCE TO THE AWE OF DONATION: THE ENIGMA IN HUSSERL'S THE IDEA OF PHENOMENOLOGY
}

\begin{abstract}
The Idea of Phenomenology is the result of the first course in which Husserl presented disciplinary developments after the transcendental turn occurred around 1905. It is therefore not only an important document of this turning, but also a high-impact work on the subsequent phenomenological tradition. In this paper we study three aspects of the category of enigma (Rätsel): the way in which it characterizes transcendence, the figure of objectors and the issue of donation and its modes. This analysis will highlight unexplored aspects of transcendental phenomenology at its early stages.
\end{abstract}

Keywords: phenomenology; Husserl; method; transcendence; donation 
LA IDEA DE LA FENOMENología es un texto pleno de tensión. Permite seguir los derroteros de una investigación amenazada por objeciones a las cuales se debe ir respondiendo mientras se insiste en la tarea de desandar prejuicios que ocultan los efectivos problemas filosóficos que la nueva disciplina está llamada a responder. En este marco presenta Husserl por primera vez la reducción fenomenológica y la idea de constitución de los objetos en la conciencia. La novedad es presentada apelando a la figura del planteo de un enigma que incentiva recorridos en vistas de una solución encontrada como recompensa a grandes esfuerzos, lo cual expresa un aspecto relevante de la impronta con que es concebida la fenomenología en este momento fundacional.

En efecto, esta estrategia para concitar atención oficia de hilo conductor y nos permitirá examinar en primer lugar su efecto en la caracterización de la trascendencia como punto que debe ser explicado para dar cuenta del conocimiento. Luego consideraremos dos ejemplos de esta estrategia que apelan a la respuesta a objetores que refuerzan la carga enigmática. Finalmente atenderemos a la presentación de la noción de dato o donación absoluta (absolute Gegebenheit), que ha tenido un importante influjo en la tradición fenomenológica posterior. Esta progresión revelará el modo en que La idea de la fenomenología nos orienta hacia un terreno fundacional donde pueden atisbarse in nuce tanto numerosos aspectos de desarrollo ulterior, que en esta primera etapa apenas están esbozados, como rasgos fundamentales persistentes de la disciplina.

\section{El hallazgo de la reducción y el conocimiento como enigma}

Los eSTUdios DE HuSSERL EN FILOSOFÍA partieron de una formación inicial en astronomía y matemáticas, primero en Leipzig, entre 1876 y 1878 , y luego en Berlín, entre 1878 y 1881, que dieron por resultado un doctorado en matemáticas en 1882 a los 23 años. La doble formación en matemáticas y en filosofía del joven Husserl dejó amplias huellas en su producción posterior. Probablemente el efecto más visible sea su preocupación temprana por los fundamentos de las matemáticas, que se extiende al problema de la legitimidad del conocimiento en general. Los enfoques contrarios al psicologismo de Bolzano dejaron sin duda 
su marca y acompañaron como un movimiento en segundo plano sus estudios en esta época. El posterior traslado a Viena propició el encuentro con Brentano, que exploraba las derivas de una psicología descriptiva encuadrada en un programa general de filosofía como ciencia rigurosa (Beyer, 1996; Benoist, 2002; Sebestik, 2003). Filosofía y estudio de la intencionalidad serían rasgos que ya no abandonarían la tarea de Husserl, de modo que el modelo de Brentano sirvió de plataforma para la exploración que caracteriza su Filosofía de la aritmética, hasta que, según declara en la Crisis, en 1898 concibió la correlación universal a priori entre objeto de la experiencia y modos de darse (Hua VI, p. 169).

El inicio de siglo alumbró la publicación del primer volumen de sus Investigaciones lógicas ${ }^{1}$, seguido en 1901 del segundo, "Investigaciones sobre fenomenología y teoría del conocimiento", orientado a estudios de psicología descriptiva. Investigaciones lógicas suele ser considerada la obra que funda la fenomenología, aun cuando representa un estadio descriptivo que habría de durar poco tiempo. Esta obra ofrece, en la introducción del segundo volumen, la fórmula de "ir a las cosas mismas" que Heidegger hizo célebre en el séptimo parágrafo de Ser y tiempo (Zirión Quijano, 1989, 2003; Xolocotzi, 2009).

En esta área avanzaron sus estudios, focalizados en torno a temas como la percepción, la memoria y la imaginación, a la par de la creciente distinción entre conciencia pura y psicología empírica que se plasma en las críticas al naturalismo, en el inicio de su estancia en Gotinga. Esta posición, así como el historicismo, ocupa el sitial de obstáculo que despeña la filosofía hacia el escepticismo y ambos constituyen el núcleo de la presentación del ensayo de 1911 "La filosofía como ciencia estricta" (Walton, 2006; Inverso, 2016a).

Los desarrollos de Investigaciones lógicas le brindaron a Husserl una rápida aceptación y un cúmulo de seguidores entusiastas, como testimonia la conformación del círculo de Múnich que acompañó sus investigaciones de estos años. Un lustro después de la publicación del primer volumen de Investigaciones lógicas, sin embargo, esta primera etapa de la fenomenología quedó clausurada en

1 El primer volumen corresponde a los "Prolegómenos a la lógica pura”, que presentan una crítica al psicologismo, usualmente asociada con las críticas que Frege le dirigiera a Filosofía de la aritmética. Sobre este punto, véanse: Hanna,1993; Mohanty, 2003; Huemmer, 2004. 
una doble encrucijada que conecta lo biográfico con lo teórico ${ }^{2}$. Por un lado, el reconocimiento creciente de su trabajo fue interrumpido por una evaluación desgraciada que frustró, al menos provisionalmente, su promoción de profesor extraordinario a profesor ordinario, cuando, en 1905, la universidad consideró que los aportes de Husserl eran científicamente insignificantes ${ }^{3}$. El traspié -uno de los varios que tuvo en su carrera académica- provocó un malestar que se ha llegado a interpretar como un desafío para probar la fertilidad de su pensamiento (Schuhmann, 1997).

Incluso si no fue así, lo cierto es que un par de meses después, en el verano de 1905 , se produjo el hito que dio lugar al surgimiento de la fenomenología trascendental, en el contexto de sus vacaciones en Seefeld, en la zona del Tirol. Un manuscrito llamativo advierte la sorpresa que deparaba esta exploración a sus discípulos Pfänder y Daubert que lo acompañaban en esos momentos. La anotación sumerge al lector en el examen del marrón de una botella de cerveza: "veo una botella de cerveza que es marrón, y me limito a lo marrón de su extensión, 'tal como es realmente dado'. Excluyo todo lo que es meramente mentado y no dado en el fenómeno" (Hua X, p. 103) ${ }^{4}$.

Se trata de la primera irrupción de la reducción que desembocaría en una redefinición completa de la fenomenología, tal como se despliega en el curso que entre abril y mayo de 1907 dictó en Gotinga y se publicó luego como La idea de la fenomenología. La relectura de Descartes constituye en este acercamiento una estrategia central para redefinir los fundamentos del método. La presencia de este rasgo hace de esta obra el primer ejemplo de descripción de un acceso hacia

2 Véanse Kuhn, Avé-Lallemant y Gladiator, 1976; Spiegelberg, 1982; Schuhmann, 1997.

3 El episodio es relatado por Husserl en los Persönliche Aufzeichnungen editados por Biemel (1956, pp. 293-294).

4 "Ich sehe eine Bierflasche, die braun ist, ich halte mich an das Braun in seinere Ausbreitung 'so wie es wirklich gegeben ist,', ich schließe alles, was im Phänomen bloßgemeint und nicht gegeben ist, aus". El manuscrito 35 o manuscrito de Seefeld sobre la individuación usualmente se consigna como un suplemento al texto de Sobre la fenomenología de la conciencia interna del tiempo. Acerca de las hojas de Seefeld, redactadas por Daubert sobre filosofía trascendental y fenomenología, véase Hamrick 1985, p. 8. 
la fenomenología en la forma de una "vía cartesiana", que resulta así el acercamiento primigenio a la fenomenología como neocartesianismo".

Este curso de cuatro horas semanales del semestre de verano de 1907 constituía una introducción a la Lección sobre la cosa, publicado más tarde como Cosa y espacio (Hua XVI). Usualmente se lo considera un texto de transición, apelando a la fecha más o menos equidistante entre las Investigaciones lógicas de 1900 y la publicación de Ideas I en 1913, pero también a cuestiones de contenido (Zirión Quijano, 1990). En Investigaciones lógicas ya está planteada la noción de la fenomenología como filosofía primera orientada al problema de la conciencia y su objeto, renegando del marco psicologista en el cual la lógica se supedita a la psicología y se pierde con ello la posibilidad de un terreno no sujeto a los vaivenes de lo empírico. La fenomenología, con su reflexión sobre los fenómenos psíquicos y su descripción de estas vivencias, resulta una ciencia ideal y ajena a la psicología como ciencia de hechos.

El objetivo de deshacerse de supuestos para examinar el objeto de la conciencia "tal como es realmente dado" está claro y ya se ensaya el análisis eidético, pero no hay en esta obra todavía una formulación explícita de la reducción fenomenológica y faltan por tanto los elementos para plantear un yo trascendental. En la quinta de las Investigaciones lógicas afirma Husserl que solo encuentra el yo empírico (Hua XIX/2, \# 8, p. 165), pero más tarde, en la segunda edición, refiriéndose al yo trascendental agrega una nota donde declara "he aprendido a encontrarlo", y sugiere que la ceguera inicial venía de la mano del temor a la metafísica del yo. En algún sentido, podríamos decir que esta nota ilustra un adelanto de lo que vendría después, precisamente en la época que retrata $L a$ idea de la fenomenología. En esta nueva etapa se verifica entre sus seguidores, tanto los contemporáneos como los actuales, la grieta entre quienes ven un giro hacia el idealismo trascendental que quiebra el ánimo de la primera fenomenología y su realismo, y quienes vislumbran una zona de continuidad en la cual se da la manifestación de formulaciones que ya estaban presentes en lecciones anteriores.

5 La cuestión de las vías hacia lo trascendental es de fundamental importancia en el despliegue del pensamiento husserliano. Sobre este punto, véase el trabajo tradicional de Kern (1977) y las relecturas de Drummond, 2005; Luft, 2004; Staiti, 2012 e Inverso, 2016 b. 
En este clima Husserl apela a crear un efecto impactante, donde la audiencia del curso primero y, posteriormente, los lectores quedan atrapados por la emoción de una trama que propone resolver un enigma. Husserl usa una treintena de veces el término Rätsel y el adjetivo rätselhaft, con una reiteración de este vocablo en lugares centrales para la argumentación, con lo cual podría decirse que La idea de la fenomenología, sobre todo en sus primeras cuatro lecciones que concentran las bases del planteo, está concebida con una tensión extrema. La apelación a las nociones de enigma y misterio enfatizan así la situación de oscuridad de la que se parte y los puntos que permitirían superarla.

\section{El enigma de la trascendencia}

EL RECORRIDO SE INICIA con la caracterización de la actitud natural como aquella que por su confianza en la realidad de lo que aparece a la conciencia es ajena a misterio alguno, porque no llega a advertirlo. La actitud natural transcurre sumida en su actividad de acumulación de juicios, relaciones contradictorias entre ellos y un complejo montaje de dispositivos para elegir en los casos conflictivos y erigir el edificio de la ciencia, todo esto sin rastros de enigma, en una transparencia ficticia que oculta los vicios de fundamento de toda la construcción. Por el contrario, la actitud filosófica nace de la percepción repentina de un misterio enraizado en la posibilidad misma del conocimiento ${ }^{6}$. La actitud filosófica queda así afectada por el enigma y ya no puede renunciar a la reflexión sobre esta esfera, dado que no hay conocimiento en el que pueda avanzarse si primero no se establecen las condiciones por las cuales es posible.

Esta actitud filosófica se identifica rápidamente con la fenomenología comprendida como "una ciencia, un conjunto de disciplinas científicas" (Hua II, p. 23$)^{7}$, en lo cual cabe señalar su unicidad, pero al mismo tiempo su función de sustrato común en el plano de la fundamentación del resto de las ciencias particulares y sus ontologías locales. Inmediatamente Husserl agrega: “[...] pero

6 Véase, por ejemplo, Hua II, p. 19, donde se dice que el conocimiento "se nos presenta de repente como un misterio [steht mit einem Mal als Mysterium da]". Sobre la caracterización de la actitud natural y la actitud fenomenológica, véase L. Rabanaque, 2011.

7 „einen Zusammenhang von wissenschaftlichen Disziplinen“. 
también designa, al mismo tiempo y por encima de todo, un método y una actitud intelectual" (Hua II, p. 23) ${ }^{8}$, poniendo de relieve la dimensión metodológica que acompaña siempre la empresa fenomenológica, que es, por tanto, a la vez una ciencia, un método y una actitud peculiar dictada por el estupor ante el enigma del conocimiento.

La definición de la actitud filosófica, marcada por la intriga ante esta situación misteriosa, se plantea inicialmente diciendo que "la donación del objeto cognoscitivo en el conocimiento, que es algo obvio para el pensamiento natural, se vuelve un enigma" (Hua II, p. 20)9 , y avanza hasta universalizarse, de modo que cualquier seguridad o confianza queda anulada:

pues la habilidad objetiva del conocimiento en cuanto tal para alcanzar un objeto se ha convertido en algo enigmático e, incluso, dudoso en lo que atañe a su sentido y su posibilidad. Y, en consecuencia, el conocimiento exacto no resulta menos enigmático que el no exacto, el científico no menos que el precientífico (Hua II, pp. 24-25) ${ }^{10}$.

Así, el misterio cubre por completo al conocimiento que queda afectado no solo en su capacidad para dar cuenta de objetos de modo adecuado, sino también en su posibilidad misma. Hacia el final de la primera lección, Descartes, todavía sin mención más que como una tradición del siglo XVII, aparece en el trasfondo como precursor de esta experiencia repentina de extrañamiento que abre a una dimensión fundante, pero opera a la vez como la señal de un fracaso. Sintetiza el intento de dotar a la filosofía de rigurosidad, pero se trata de un proyecto incompleto y que, por ello, derivó en la confusión de equiparar la filosofía con otras ciencias. E incluso en algo todavía peor: el intento de basarla en otras ciencias, como la psicología y la biología, con lo cual esta línea habría olvidado que las ciencias pueden apoyarse unas en otras, pero esto no le sucede a la filosofía, a

8 "bezeichnet aber zugleich und vor allem eine Methode und Denkhaltung".

9 „Die dem natürlichen Denken selbstvertändliche Gegebenheit der Erkenntnisobjekte in der Erkenntnis wird zum Rätsel“.

10 „Denn objektive Triftigkeit der Erkenntnis überhaupt ist nach Sinn und Möglichkeit rätselhaft und dann auch zweifelhaft geworden, und exakte Erkenntnis wird dabei nicht minder rätselhaft als nicht exakte, wissenschaftliche nicht minder als vorwissenschaftliche". 
riesgo de perder sus rasgos propios. En ambos casos, el estupor del enigma produce la necesidad de buscar puntos de partida totalmente nuevos (Hua II, p. 24).

Esta novedad adviene en la segunda lección, que presenta la estrategia de "adjudicar el índice de la 'dubitabilidad' al mundo entero" (Hua II, p. 29) ${ }^{11}$, delineando la epoché como el elemento que orienta hacia un conocimiento dado absolutamente libre de incertidumbre. La condición de posibilidad del conocimiento primario es, entonces, que esté libre de la "vaguedad e incertidumbre que por lo general presta a los conocimientos ese carácter enigmático y problemático" (Hua II, p. 29) $)^{12}$. Las expectativas sobre este recurso se advierten claramente en la siguiente consideración:

Si no nos está permitido tomar como dado previamente ningún ser, porque la falta de claridad gnoseológica trae consigo que no comprendamos qué sentido puede tener un ser que sea en síy que, no obstante, sea conocido en el conocimiento, entonces ha de ser posible mostrar un ser que podamos reconocer como dado en forma absoluta y exento de cualquier duda en la medida en que está dado con completa claridad, sobre cuya base toda pregunta encuentre y haya de encontrar su respuesta inmediata (Hua II, pp. 29-30) ${ }^{13}$.

En efecto, la epoché no está puesta, como en la tradición más antigua, al servicio del escepticismo, sino que apunta a poner de relieve un punto seguro, para lo cual se impone restringir el conocimiento a aquellos datos absolutos totalmente evidentes ${ }^{14}$. La solución del enigma pide un pilar de evidencia imposible de encontrar en los inasibles objetos de la trascendencia. La mención que Husserl

11 „die ganze Welt [...] die sich mit dem Index der Fraglichkeit zu verstehen“.

12 „Unklarheit und Zweifelhaftigkeit, die erkenntnissen sonst den Charakter des Rätselhaften, Problematischen verleihen".

13 „Wenn wirkein Sein als vorgegeben hinnehmen dürfen, weil die erkenntniskritische Unklarheit es mit sich bringt, das san sich und doch in der Erkenntnis erkannt sei, so miss sich doch ein Sein aufweisen Lassen, das wir als absolut gegeben und zweifellos anerkennen müssen, sofern es eben in einer weise gegeben ist, dass bei ihm völlige Klarheit besteht, aus der Frage ihre unmittelbare Antwort findet und finden muss".

14 Sobre la noción y usos de epoché en la tradición previa, véase Inverso, 2015, especialmente lo pertinente a la tradición antigua y a Descartes. 
trae a cuento, ahora explícitamente, para enfocar este punto es la meditación cartesiana sobre la duda, señalando que el error puede llevar a la "desesperación escéptica [skeptische Verzweiflung]", pero que puede ser contrarrestada con la evidencia surgida de que es indudable que juzgo y que por tanto la duda universal resulta absurda (Hua II, p. 30).

Lo mismo se aplica a toda cogitatio, de modo tal que con las modificaciones debidas se puede usar esta consideración cartesiana, puesto que toda vivencia intelectual es un dato absoluto y desde el inicio puede indicarse una esfera de donación absoluta. Que estemos frente al plano de una absolute Gegebenheit implica que no depende de ningún elemento condicionado y funciona por eso como el punto de partida buscado. Husserl no ahonda aquí en los aspectos de la argumentación cartesiana que deben ser alterados, pero es claro que la construcción de la figura de Descartes como una iniciación imperfecta reverbera en la tradición fenomenológica posterior a través de distintos diagnósticos de falla, de un modo que permitiría construir un mapa de posiciones y tensiones fenomenológicas a partir del modo en que cada una diagnostica el error de Descartes. Un recorrido por las filosofías de Henry y Marion y las líneas asociadas con el giro teológico, así como las más recientes vinculadas a los nuevos realismos, muestran esta actitud de modo persistente.

La crítica del conocimiento debe partir, por tanto, de lo que se da de modo indubitable y por tanto escapa "al enigma que había sido el motivo de todas las confusiones escépticas” ( Hua II, p. 33) ${ }^{15}$. Con esto se sugiere que el escepticismo íntegro es una desviación propia de la actitud natural y que desaparece con la epoché. Este no es más que el inicio del planteo, que avanza con tensión creciente, dando lugar a la intervención de objetores posibles con preguntas peligrosas que amenazan con hacer fracasar los avances. El clima que construye Husserl es precisamente uno de alta tensión dramática, trazando el argumento con la aparición de un enigma, un avance costoso en medio de la perplejidad y adversarios que amenazan el éxito de la tarea. Los objetores encarnan la serie de equívocos de la tradición filosófica previa y disparan objeciones que, en rigor, se revelan como sofismas.

„von dem Rätsel, das den Anlass zu allen skeptischen Verwirrugen gegeben hatte“. 


\section{Portadores del enigma: la apelación a los objetores}

LA CATEGORÍA DE ENIGMA está especialmente presente en dos casos que podemos tomar como ejemplos. En el primero, Husserl se pregunta sobre la posibilidad misma del conocimiento. Según este tipo de objeción, el cuestionamiento solo es radical si parte de la siguiente condición: "si para la teoría del conocimiento todo conocimiento es un enigma, entonces también lo habrá de ser el primero con el que ella misma comience" (Hua II, p. 34$)^{16}$.

Este objetor toma la epoché como procedimiento que afecta a todo conocimiento y no solo a la tesis de efectividad del mundo, con lo cual anula la posibilidad misma de conocer, de un modo que recuerda las paradojas megáricas reflejadas en el pasaje platónico de Menón (80d-e), por el cual todo intento de conocimiento queda paralizado: no le es posible a nadie buscar ni lo que sabe ni lo que no sabe, pues ni podría buscar lo que sabe - puesto que ya lo sabe, y no hay necesidad alguna entonces de búsqueda-, ni tampoco lo que no sabe -puesto que, en tal caso, ni sabe lo que ha de buscar- ${ }^{17}$. Del mismo modo que Platón muchos siglos antes buscó una salida a esta paradoja, Husserl debe excluir toda interpretación en la que el ejercicio de la epoché se malentienda y cancele la posibilidad misma de un conocimiento primario cierto. Para ello sostiene que en esta pretendida proyección universalizante hay engaño, pero se disipa al advenir ciertos conocimientos que se muestran indubitables. Cifrando en la reducción la salida a la objeción escéptica, el conocimiento en cuanto tal pierde su carácter enigmático, precisamente porque "el enigma no habita en ciertos otros conocimientos" (Hua II, p. 34) ${ }^{18}$.

Se intenta, por tanto, señalar el terreno firme que emerge en el marco de lo dudoso. Cabe señalar que el desafío del objetor que quiere extremar el desconcierto permite delinear el núcleo del enigma, es decir perfilar los límites que separan lo cierto de lo dudoso, aclarando que lo que provoca perplejidad es el

16 „und ist der Erkenntnistheorie alle Erkenntnis ein Rätsel, so auch die erste, mit der sie selbst beginnt“.

17 Sobre la paradoja del conocimiento, véase Nehamas, 1985 y Canto-Sperber, 1991; y, en relación con las críticas platónicas en contra de los megáricos, Inverso, 2014. 
plano de la trascendencia. Esta situación se produce porque, saliendo del plano de la inmanencia, se intenta afirmar "conocimiento trascendentemente objetivante" (Hua II, p. 34) ${ }^{19}$, es decir que "pone objetos como existentes" (Hua II, p. 35$)^{20}$ sobre los que no se tiene seguridad alguna. Si este desvío no se produce, el conocimiento está a salvo.

El segundo ejemplo de desafío parte de estos resultados y los explicita. Aparece poco después en la misma segunda lección, donde se distinguen los sentidos de inmanencia y de trascendencia para afirmar que la parte ingrediente en el acto de conocimiento es evidente, mientras que lo que no está así contenido resulta enigmático. Husserl nota que todo lo perteneciente a la trascendencia en cualquiera de sus sentidos es problemático, porque "es el enigma que se cruza en el camino del conocimiento natural y constituye el impulso para las nuevas investigaciones" (Hua II, p. 36) ${ }^{21}$.

Esto lo lleva a resumir la tarea de la crítica del conocimiento en términos de dar solución al problema de la trascendencia, sintetizado como "nada trascendente puede emplearse como dado previamente" (Hua II, p. 36) ${ }^{22}$. Así, hay que determinar ahora ya no que es posible, sino cómo es posible el conocimiento. En este contexto se desarrolla una respuesta a un posible objetor que, al contrario del anterior, que con aires escépticos despeñaba todo intento en el fracaso, minimiza el problema y con un optimismo ingenuo quiere utilizar los datos trascendentes para responder a esta cuestión. Este objetor plantea que "lo enigmático es el cómo, mientras el qué es absolutamente seguro" (Hua II, p. 36) ${ }^{23}$, dando por sentado que hay conocimiento y que la misma práctica de relación con el mundo refuta la pregunta por su posibilidad, disolviendo cualquier duda escéptica al respecto.

19 "transzendent objektivierende Erkenntnis“.

20 „setzt Objekte als seiend“.

21 „sie ist das Rätsel, das der natürlichen Erkenntnis in den Weg tritt und den Antrieb für die neuen Forschungen bildet".

22 „es darf danach Transzendentes nicht als vergegeben benützt werden“.

23 "das Wie ist rätselhaft, während das Dass absolut sicher ist". 
Se trata de una posición de amplios antecedentes, cuyo exponente más famoso es sin duda la impugnación aristotélica al objetor del principio de no contradicción en la Metafísica, (IV.4, 1008b1-30). El último de la serie de argumentos consiste precisamente en desconocer la legitimidad del cuestionamiento mismo, apelando a que en el trato con el mundo el objetor mostraría que la duda es ficticia y solo fruto de un deseo de discutir. En el caso aristotélico el negador del principio de no contradicción se detiene frente al precipicio y en su praxis le confiere al objeto el grado de identidad y univocidad que debiera negarle si realmente dudara de tal principio. El objetor husserliano diría, del mismo modo, que la práctica científica en su conjunto opera sobre la aceptación del conocimiento, por lo cual esta praxis anula cualquier duda acerca de que este es posible y limita la duda al cómo, anulando de este modo la crítica radical del conocimiento y transformándola en una epistemología que no se diferencia en estatus del resto de las ciencias, salvo por su objeto específico.

El modo de responder consiste en embarcar este intento en un callejón sin salida, porque el objetor debería afirmar el conocimiento trascendente e inferir de ello que, dado que hay conocimiento de lo trascendente, entonces hay conocimiento. Esto último se vuelve un enunciado analítico y nada dice sobre el cómo, pues sin la intuición de la unidad entre conocimiento y objeto de conocimiento la separación es tal que toda explicación es imposible y un personaje así debería concluir que su confianza en la captación de lo trascendente es un prejuicio (Hua II, p. 38).

Estas dos objeciones hacen posible afirmar que la peculiaridad de la filosofía que adopta la fenomenología como su método radica en su carácter último tantas veces desdibujado con el objetivo de alcanzar una estabilidad por medios equivocados. Distinguiendo el carácter de lo enigmático, la pregunta será ahora cómo valerse de los datos de la inmanencia y cómo acceder a objetos trascendentes para, de ese modo, establecer una correspondencia entre ellos y el acto de conocimiento. La intencionalidad apunta a dar cuenta de este acceso y de la peculiar correspondencia entre los componentes reales e intencionales de la conciencia ${ }^{24}$.

24 Sobre la intencionalidad y su función en el sistema general, véanse Mohanty, 2005; Drummond, 2005 y Walton, 2009. 


\subsection{DONACIÓN ABSOLUTA Y HUELLAS DE PLATONISMO}

EN EL INICIO DE LA TERCERA LECCIÓN se resumen las dos primeras aludiendo a la trascendencia como enigma y a la prohibición de tomar por real lo trascendente (Hua II, p. 43). La distinción entre las esferas de la inmanencia y la trascendencia permite a Husserl enfatizar ahora que en ningún caso está permitido tomar en consideración la realidad de lo trascendente; por el contrario, es preciso tomar en cuenta la esfera de las cogitationes, que "está libre del enigma de la trascendencia" (Hua II, p. 43) ${ }^{25}$. Afirma entonces respecto de las vivencias que "representan una esfera de datos inmanentes absolutos, en cualquier sentido en que interpretemos inmanencia" (Hua II, p. 43, el énfasis es nuestro) ${ }^{26}$, es decir, ya se trate de la inmanencia ingrediente (reell), que apunta a los componentes inherentes y constitutivos de un acto o una vivencia, o de los componentes reales (real) de un acto psicológico ${ }^{27}$. La reducción investiga los primeros, sin confundir fenómeno puro con fenómeno psicológico, siendo este último el que se da atado a un yo empírico como una cosa del mundo. Con este reparo, y por medio de la reducción fenomenológica, se puede partir de un fenómeno psíquico y acceder a un fenómeno puro de modo tal que se revele en la inmanencia de la conciencia como dato absoluto (absolute Gegebenheit) (Hua II, p. 45).

El avance en la resolución del enigma reposa en este punto en la figura de Descartes, que como hemos visto aparece primero apenas aludida, luego mencionada en su apelación a la duda en la segunda lección y retomada en la tercera lección precisamente porque anticipó la pregunta sobre los fundamentos de esta donación de evidencia de la vivencia. Esto sugiere que el recorrido husserliano es

25 „ist vom Rätsel der Transzendenz frei“.

26 „absoluter immanenter Gegebenheiten darstellen, in welchem Sinn wir Immanenz auch deuten“.

27 Seguimos a Zirión Quijano, que traduce reell como "ingrediente", para diferenciarlo de "real" (real), aplicado a lo que existe de modo espacial o temporal, frente a lo ideal o lo irreal, sustraídos al tiempo. Reell refiere a lo que se encuentra efectivamente contenido en las vivencias intencionales como sus partes. Frente a ello, para mentar las cosas del mundo, Husserl se vale de los adjetivos real o wirklich. Los contenidos reales (reell) se diferencian de los contenidos ideales (ideell) de la conciencia, que refieren a lo que la conciencia mienta, el objeto meramente intencional, noemático. Sobre este punto, véanse $\$ 14$ y $\$ 16$ de la quinta de las Investigaciones lógicas (Hua XVIII). 
una especie de versión que redobla la profundidad del planteo, dado que comprende el cogito de modo más completo y por tanto los criterios de claridad y distinción atestan y legitiman lo que se da en una vivencia "en un sentido más puro”. De ese modo evita sobre todo la repetición del error cartesiano, en el cual las meditaciones tercera y cuarta habilitan de modo indebido un supuesto acceso cognitivo a lo trascendente (Hua II, pp. 49-51).

Con esta redefinición del camino cartesiano queda planteado que la percepción no alcanza lo trascendente, sino lo inmanente "en la forma de la percepción refleja y puramente inmanente" (Hua II, p. 49) ${ }^{28}$, es decir reducida. En este sentido, el esquema cartesiano de correspondencia se complejiza, ya que no se trata de acto mental y objeto extramental, sino que la conciencia realiza múltiples operaciones de constitución a las que Husserl prestará atención creciente y llevarán al análisis en términos de nóesis y nóema en Ideas I. De este modo, concluye Husserl, tiene sentido dudar de un fenómeno que mienta algo que no está dado en el fenómeno, pero no si al intuir no se mienta nada más que lo aprehendido en la intuición (Hua II, p. 49).

La amplitud de este ámbito de vivencias se enfatiza en el planteo según el cual, en el régimen de reducción, se dan con autodonación absoluta no solo objetos singulares, sino también universales, lo cual abre al análisis de esencias. La fenomenología trata, entonces, "con el a priori en la esfera de los orígenes, de las donaciones absolutas" (Hua II, p. 52) ${ }^{29}$. Se ocupa de especies y estados a priori que se constituyen sobre esas especies de manera inmediatamente intuíble. Con ecos platónicos, Husserl plantea que los objetos intencionales tienen un eidos, una esencia, de modo que junto a los objetos particulares adviene la esencia; junto a los colores particulares instanciados en las cosas adviene la esencia 'color', que no surge de una construcción a partir de datos empíricos, sino por intuición eidética.

Por este planteo se acusó a Husserl de platonismo ${ }^{30}$. Cabe aclarar, sin embargo, que nunca se confiere estatus alguno a las ideas fuera de la conciencia y lo

28 „in Form der reflektiven un rein immatenten“.

29 „mit dem A priori in der Sphäre der Ursprünge, der absoluten Gegebenheiten“.

30 Husserl respondió a esta crítica en la segunda edición de Investigaciones lógicas, remitiendo los antecedentes a las "verdades en sí mismas" planteadas por Bolzano y la interpretación de la doctrina 
inteligible se forma a partir de lo sensible, único plano real, frente a la idealidad de los universales, más un excedente que se revela en el hecho de que no todas las formas categoriales se plenifican en una intuición sensible. Esto habilita el planteo acerca de la intuición categorial, que complejiza la explicación de la experiencia, donde se incluyen objetos concretos, pero también abstractos imprescindibles para dar cuenta de la experiencia efectiva.

El ámbito de la intencionalidad surge en el inicio de la cuarta lección. Allí señala Husserl que "pertenece a la esencia de las vivencias cognoscitivas tener una intentio" (Hua II, p. 55) ${ }^{31}$, es decir que las vivencias siempre mientan algo, se refieren a una objetualidad, que permanece ajena. De este modo, "lo objetual puede aparecer, puede tener cierta donación en su aparecer, si bien no está como ingrediente en el fenómeno cognoscitivo ni existe como cogitatio" (Hua II, p. 55) ${ }^{32}$. La indicación de su carácter no ingrediente implica que el objeto intencional es independiente del objeto externo, dado que la intencionalidad es un rasgo intrínseco de la conciencia, que no opera con representaciones. En este sentido, el conocimiento investiga lo inmanente ingrediente y lo inmanente en sentido intencional.

La fenomenología procede aplicando la reducción y en ese marco se atiene a la intuición pura, lo cual constituye la metodología propia de la filosofía y de la crítica del conocimiento en tanto punto fundamental orientado al cuestionamiento de supuestos. Con esta afirmación y establecimiento de vínculos, este entramado se aplica a la crítica de la razón en general, lo cual, por un lado, muestra la amplitud de los alcances, dado que atañe "también a la crítica de la razón valorativa y práctica". Por otro, asocia este tratamiento del enigma al que tematiza mucho después, en la Crisis, el enigma de todos los enigmas y lo identifica

platónica de Lotze, que reniega de todo sustancialismo de las entidades ideales, que Husserl juzga una posición superada. Véanse, por ejemplo, Hua XX/I, p. 121 y Hua XXIV, p. 37. Sobre el platonismo de Husserl, véase A. de Muralt, 1974, pp. 35-43.

31 „die Erkenntniserleibnisse, das gehört zu ihrem Wesen, haben eine intentio“.

32 „das Gegenständliche kann erscheinen, kann im Erscheinen eine gewisse Gegebenheit haben, während es gleichwohl weder reell im Erkenntnisphänomen ist, noch auch sonst als cogitatio ist". 
precisamente con la razón ${ }^{33}$. El enigma del conocimiento es la trascendencia y el enigma de todos los enigmas es la razón, como función de la conciencia en tanto maravilla de todas las maravillas.

Cuando hay intuición pura, es posible que la conciencia aprehenda su objeto directamente y por tanto tenga evidencia, ya que "evidencia no significa otra cosa que autodonación adecuada”. Con esta aclaración resulta que, si bien hemos visto que la trascendencia arrastra el enigma del conocimiento, esto no implica que la fenomenología y su estrategia reductiva se limiten a la inmanencia delineada en la cogitatio, sino que esta reconducción instala en primer plano "la esfera de la autodonación pura" y su evidencia. La noción de autodonación refuerza un punto que será importante en la tradición posterior y nota que el aparecer se da "desde" el fenómeno, dado que el fenómeno es lo que se muestra y, en términos marionianos, se muestra porque se da. Husserl afirma a propósito de esto que "la donación absoluta es algo último" (Hua II, pp. 7 y ss.), entendiendo por ello que no depende de ninguna mediación. De este modo, negar la autodonación es declarar todo ilusión y caer en el contrasentido del escepticismo ${ }^{34}$. Este énfasis está puesto a los efectos de señalar que autodonación pura implica manifestación completa, "lo que está dado exactamente en el sentido que está mentado, y dado por sí mismo en el sentido más estricto” (Hua II, p. 61) ${ }^{35}$. Por ello, evita todo peligro de contaminar la evidencia pura con evidencia mediata.

La donación absoluta, así entendida, no significa que haya autodonación de objetos singulares ingredientes, y Husserl plantea por ello la cuestión de la

33 Afirma Husserl en la Crisis: "Cada vez más se vuelve enigmática la razón misma y lo existente para ella [...], hasta que finalmente el problema universal de la más profunda relación esencial entre la razón y lo existente en general, que se hizo conscientemente manifiesto, debió convertirse en el enigma de todos los enigmas (Rätsel aller Rätsel), en el verdadero tema de la filosofía (Hua VI, p. 208)". Esta expresión hiperbólica recuerda otra bien conectada a esta, que presenta "la maravilla de todas las maravillas": el puro ego y la pura conciencia, en Ideas III (Hua $V$, p. 75), fórmula a la que Heidegger responde con "que las cosas existan", en el postfacio a ¿Qué es metafísica? (GA, p. 9).

34 Este punto explica la recurrencia de la línea de recepción en que se encuentran, por ejemplo, Michel Henry y Jean-Luc Marion respecto de este texto, en el cual Husserl vuelve sobre la noción de donación para aclarar que la reducción fenomenológica no implica limitarse a la esfera de la cogitatio, sino que refiere a la esfera de la autodonación pura. „was genau in dem Sinn, in dem es gemeint ist, auch gegeben ist und selbstgegeben im strengsten Sinn“. 
extensión de la autodonación y la pregunta por el sentido en que se vincula con las cogitationes y los objetos universales que las generalizan. A propósito de esto, Husserl aclara que no se trata de tomar las vivencias absolutas como material de mecanismos de abstracción intuitiva, dado que con esto crece exponencialmente el riesgo de introducir elementos no certificados y reanimar lo enigmático. Es por ello que se plantea que, si bien es cierto que el "conocimiento intuitivo es aquella forma de razón que se propone traer el entendimiento a la razón” (Hua $I I$, p. 62$)^{36}$, debe hacerlo con lo menos posible de entendimiento para que no comprometa y distorsione con su falta de garantía.

Se establece de este modo un planteo que se repetirá en toda la obra husserliana y que tendrá especial impacto en la crítica al psicologismo. Este yerra precisamente porque confunde la investigación intuitiva con la esfera de la percepción interna ${ }^{37}$. Hay múltiples modos de objetualidad y con ellos múltiples modos de donación, y la donación del ser que se da en la percepción interna se asocia con la de la ciencia natural. En obras posteriores extraerá Husserl más consecuencias de esta asociación para indicar la radical diferencia de la fenomenología.

\subsection{LOS MODOS DE LA DONACIÓN}

LA QUINTA LECCIÓN constituye un texto especialmente influyente en la fenomenología posterior. De la rápida mención del modo en que de la percepción del color se obtiene, por reducción, el fenómeno puro de color, y, por abstracción pura, la esencia de color fenomenológico en cuanto tal, Husserl pasa al examen del recuerdo, donde parece suceder algo similar, pero le interesa notar que en la captación del tono de un fenómeno sonoro parece captarse con evidencia más allá del ahora, de modo que el recuerdo retiene intencionalmente la fase temporal (Hua II, pp. 67-68). El ejemplo de la duración del tono sonoro sirve para señalar que lo temporal ofrece el caso de algo que no se encuentra en el fenómeno

36 "Schauende Erkenntnis ist die Vernunft, die sich vorstzt, den Verstand eben zur Vernunft zu bringen“.

37 Sobre la cuestión del psicologismo, véanse T. Seebohm, 1991, pp. 149-182; D. Jacquete, 2003, y especialmente J. Mohanty, 2003, pp. 113-130, y M. González Porta, 2010. 
"y aun así se constituye en el fenómeno" (Hua II, p. 68) ${ }^{38}$. Por esta vía se acerca Husserl a la donación de esencias y su función de universalización, de lo cual son ejemplo la rememoración y la fantasía tanto como la percepción. En todos los casos hay intuición y las posiciones de existencia son irrelevantes, de lo cual se ofrecen como ejemplos los casos de la fantasía sin recuerdo y el color fantaseado, que muestran que el juicio de esencias es independiente de la diferencia entre percepción y fantasía (Hua II, pp. 69-70).

Esto permite afirmar que existencia y esencia son modos de ser en dos modos de autodonación y lo que interesa al análisis fenomenológico es el juicio genérico de esencias que excede a los objetos singulares. La evidencia de la cogitatio no es algo simple, sino que abre a un amplio plexo de apariciones que crean los objetos. En este sentido dirá Husserl que "la conciencia que intuye es [...] actos de pensamiento formados de ciertas maneras" (Hua II, p. 72) 39 para que haya donación de las cosas. Por ello se sugiere que "hasta donde llegue la evidencia real efectiva, hasta allí llega también la donación” (Hua II, p. 73) $)^{40}$, pero hay que averiguar qué está verdaderamente dado y qué no, con el objetivo, de nuevo, de evitar introducir los efectos de pensamiento inauténtico.

Esto pone a Husserl en la necesidad de mostrar los diferentes modos de la donación auténtica, que indica "la constitución de los diferentes modos de objetualidad y las relaciones de unos con otros" (Hua II, p. 74) ${ }^{41}$. Donde podríamos esperar una lista exhaustiva adviene una enumeración abierta:

[...] la donación de la cogitatio, la donación de la cogitatio revivida en un recuerdo fresco, la donación de la unidad de la aparición que dura en la corriente de fenómenos, la donación de su modificación, la donación de la cosa en la percepción "externa", la donación de las diferentes formas de la fantasía y la rememoración, así como, en los nexos correspondientes, la donación de múltiples percepciones y demás formas de representación que se unifican sintéticamente.

38 „und doch konstituiert es sich im Phänomen“.

39 "das schauende Bewusstsein, das sind [...] so und so geformte Denkakte“.

40 „soweit wirkliche Evidenz reicht, soweit reicht Gegebenheit“.

41 „die Konstitution der verschiedenen Modi der Gegenständlichkeit und ibre Verhältnisse zueinander berauszustellen". 
Y, por supuesto, también tenemos las donaciones lógicas, la donación de la universalidad, del predicado, del estado de cosas, etcétera; y asimismo la donación del contrasentido, de la contradicción y del no-ser, etc. (Hua II, p. 74).

Tras este listado Husserl sostiene que la donación se da en el fenómeno del conocimiento, y es por ello que "en la consideración de esencias hay que investigar por todas partes esta correlación por el momento tan maravillosa" (Hua $I I$, p. 74$)^{42}$, en un asombro que es en algún sentido el corolario de la salida del enigma. Podría decirse que el misterio de la trascendencia lleva a la maravilla de la donación. En esta clave debe comprenderse la afirmación de que "el problema verdaderamente importante es el de la donación última de sentido del conocimiento y, por tanto, el de la objetualidad en cuanto tal, que solo es lo que es en su correlación con el conocimiento posible" (Hua II, pp. 75-76) ${ }^{43}$.

Solamente en este marco puede intuirse con evidencia. La donación, por ser absoluta, ofrece la norma última.

Con la vinculación entre la correlación de conocimiento y objetualidad y la donación concluye el texto de las lecciones, pero cabe detenerse en otra dimensión de esta misma obra, especialmente porque se revela de suma importancia para nuestro recorrido. El texto de La idea de la fenomenología se estableció a partir del manuscrito y la transcripción de Landgrebe, hecha entre 1923 y 1926. Contiene en escritura taquigráfica el texto de las lecciones, pero adosa también un curso de ideas que parece haber sido una reformulación para la quinta lección que resume los puntos salientes del curso íntegro. En este sentido, sirve como una visión comprehensiva que ordena lo que hemos visto y marca tres estratos de reflexión que introducen a la fenomenología, lo cual hace del "Curso de ideas" un recorrido en sí mismo relevante.

Allí se sintetiza la temática diciendo que trata de cómo se alcanza el conocimiento de "las cosas que existen en sí mismas [an sich Seienden Sachen]" (Hua $I I$, p. 3). En efecto, partiendo de la distinción entre actitud natural y actitud

42 „in der Wesenbetrachtung dieser zunächst so wunderbaren Korrelation nachzugehen“.

43 „das wabrhaft bedeutsame Problem das der letzten Sinngebung der Erkenntnis ist und damit zugleich der Gegenständlichkeit überhaupt, die nur ist, was sie ist, in ihrer Korrelation zur möglichen Erkenntnis“. 
filosófica, afirma que al reflexionar de modo natural sobre el conocimiento se cae en contrasentidos, por lo cual se debe avanzar en su crítica siguiendo el método fenomenológico entendido como "doctrina universal de las esencias, en la que la ciencia de la esencia del conocimiento se encuentra en su lugar" (Hua II, p. 3) $)^{44}$. Entre ellas, la filosofía debe desentrañar la esencia misma del conocimiento como condición de posibilidad para la validez de cualquier afirmación ulterior.

El método que este objetivo supone se resume en tres pasos que aquí mencionamos brevemente para señalar los puntos que Husserl considera centrales en el camino recorrido y organizan, por tanto, su visión del método en este momento. El primero, como hemos visto, parte de la meditación cartesiana sobre la duda y ofrece el dato incontrovertible de la cogitatio en tanto dato absoluto (absolute Gegebenheit). El término Gegebenheit apunta aquí a dar cuenta de lo que es dado de manera directa a la conciencia y es por tanto ya un conocer, aspecto que, según vimos, cierra la quinta lección. Con la renuncia a lo trascendente, es preciso operar sobre el material de las vivencias a través de la reducción fenomenológica, que implica precisamente una suspensión de las posiciones trascendentes sin pérdida de contenido, ya que se accede a todas las ciencias como fenómenos y no como sistemas de verdades.

Sobre esta base, en el segundo paso en la consideración fenomenológica se produce un abandono de la psicología, a la vez como ciencia empírica, a la manera de las críticas de Investigaciones lógicas, y como psicología descriptiva, orientada a vivencias que conservan el sentido de existentes en el mundo. No se trata de cómo puede el yo mundano alcanzar en sus vivencias un ser en sí, sino "cómo puede la absoluta autodonación del conocimiento alcanzar algo que no se da en sí mismo" (Hua II, p. 7) ${ }^{45}$. Con la referencia a la abstracción ideativa, la intuición de esencias, se abre el campo de la objetividad esencial. La reducción fenomenológica excluye lo trascendente y revela un campo de lo a priori dentro de la autodonación absoluta, donde todas las cosas, incluidas "yo, el mundo y

\footnotetext{
44 "die allgemeine Wesenslehre, in die sich die Wissenschaft vom Wesen der Erkenntnis einordnet“. 
Dios, así como las multiplicidades matemáticas y las objetividades científicas, permanecen en la indecisión" (Hua II, p. 9) ${ }^{46}$.

Se trata de un punto fundamental en la presentación, ya que bosqueja una noción de sujeto bien distinta de la tradición cartesiana, a la que refiere Husserl diciendo que "para Descartes, descubrirla y abandonarla fue todo uno" (Hua II, p. 10). Reparemos en este punto en la apelación al error cartesiano para aludir a la necesidad de evitar el psicologismo, lo cual abre un vínculo entre las referencias a la psicología y la reelaboración del cartesianismo, que en la visión tradicional sobre las vías hacia la fenomenología aparece oscurecido.

Para concluir este curso de ideas, Husserl presenta el tercer paso en la consideración fenomenológica, indicando un tercer estrato de reflexión, a propósito de la extensión y límites de la autodonación, dado que lejos de un esquema simple, donde se captan los propios actos y se eleva lo intuido a conciencia de lo universal, adviene la amenaza de que las cogitationes "ocultan todo tipo de trascendencias” (Hua II, p. 11) ${ }^{47}$. La ambigüedad del fenómeno apunta al fenómeno mismo y a lo que aparece en él, ofreciendo dos datos absolutos, donde el objeto no es un fragmento del fenómeno.

Con esto se presenta el problema de la constitución, que apunta a que las cosas se constituyen en las vivencias, aunque no puedan encontrarse como ingredientes en aquellas vivencias: son y existen en la aparición y están dadas en sí mismas gracias a ella. La fenomenología investiga los tipos de correlación entre fenómeno del conocimiento y objeto de conocimiento, de modo tal que el objeto real trascendente se constituye gradualmente. Este camino lleva a plantear que se trata de problemas de la donación, entendidos como "problemas de la constitución de todo tipo de objetualidades en el conocimiento" (Hua II, p. 14) ${ }^{48}$, aclarando a la vez que no se trata de toda autodonación, sino de la que opera un yo trascendental tras la reducción, de modo tal que emerge el método del análisis de esencias en la esfera de la evidencia inmediata.

\footnotetext{
46 "das Ich und Welt und Gott und die mathematischen Mannigfaltigkeiten und was immer für wissenschaftliche Objektivitäten dabingestellt bleiben".

47 „bergen allerlei Transzendenzen“.

„der Konstitution von Gegenständlichkeiten jeder Art in der Erkenntnis“.
} 
4. Corolarios

EL INTERÉS PRIORITARIO DE LA TRAMA de La idea de la fenomenología es sentar la posibilidad de una crítica fenomenológica del conocimiento mostrando sus requisitos y en este sentido no es una aplicación del método sino una presentación de su plausibilidad. E esta obra conviven un nivel crítico que apunta al problema de la posibilidad del conocimiento y un nivel meta-crítico que explora el problema de "la posibilidad del conocimiento de la posibilidad del conocimiento" con ambos planos en interacción. En este marco se presenta la relación entre el material cartesiano y el desarrollo fenomenológico de un modo que seguiría el propio camino de descubrimiento por el que avanzó Husserl, como sugiere L. Hardy (1999, p. 3).

El recurso al cartesianismo que hemos encontrado y que debe ser redefinido para convertirlo en herramienta de resolución del enigma de la trascendencia apela a la autodonación inmediata de la conciencia y su diferencia con la donación de los objetos, de modo que la primera se da plenamente y aparece de forma total, mientras que la segunda es siempre parcial. La donación inmanente de la conciencia constituye por tanto un campo que resiste a toda duda y ofrece el suelo firme que es imposible encontrar en los objetos trascendentes. No encontramos en esta obra todavía las precisiones ni los enfoques de detalle que caracterizan Ideas $I$, pero los anuncia anticipadamente, ofreciendo el fundamento de las argumentaciones. Con ello arribamos, habiendo pasado del enigma de la trascendencia a la maravilla de la donación, al terreno de la fenomenología como ciencia eidética descriptiva de las vivencias de la conciencia trascendentalmente reducidas.

\section{Referencias}

Benoist, J. (2002). Husserl and Bolzano. En: A.T. Tymieniecka. (Ed. de la serie). Analecta Husserliana. The Yearbook of Phenomenological Research: Vol. 80. Phenomenology World-Wide (pp. 98-100). doi:10.1007/978-94-007-04732_8

Beyer, C. (1996). Von Bolzano zu Husserl. Eine Untersuchung über den Ursprung derphänomenologischen Bedeutungslehre. Dordrecht: Kluwer. 
Biemel, W. (1956). Husserl, Persönliche Aufzeichnungen. Philosophy and Phenomenological Research, 16(3), 293-302.

Canto-Sperber, M. (1991). Traduction inédite, introduction et notes. Platon, Ménon. Paris: Flammarion.

De Muralt, A. (1974). The Idea of Phenomenology. Illinois: Northwestern University Press.

Drummond, J. (2005 [1975]). The Structure of Intentionality. En: R. Bernet, D. Welton, \& G. Zavota. (Eds.). Critical Assessments of Leading Philosophers (vol. 3) (pp. 31-60). London: Routledge.

Follesdal, D. (1978). Brentano and Husserl on Intentional Objects and Perception. Grazer Philosophische Studien, 5, 83-94.

González Porta, M. (2010). Psicologismo e idealismo en Frege y Husserl. Capa, $117(37), 57-86$.

Hamrick, W. (1985). Phenomenology in Practice and Theory. Dordrecht: Martinus Nijhoff.

Hanna, R. (1993). Logical Cognition: Husserl's Prolegomena and the Truth in Psychologism. Philosophy and Phenomenological Society, 53, 251-275.

Hardy, L. (1999). The Idea of Phenomenology. Dordrecht: Kluwer.

Heidegger, M. (1976). [GA]. Gesamtausgabe, 9, Wegmarken. F.-M. von Herrmann (Ed.). Frankfurt am Main: Vittorio Klostermann. [Hay edición en castellano: Heidegger, M. (2007). Hitos. (A. Leyte y H. Cortés, Trad.). Madrid: Alianza].

Husserl, E. (1950-2009). [Hua] Husserliana: Gesammelte Werke I-LX. Dordrecht: Springer [con anterioridad: Den Haag: Martinus Nijhoff; Dordrecht/ Boston/London: Kluwer Academic Publishers].

Huemer, W. (2004). Husserl's Critique of Psychologism and his Relation to the Brentano School. En: A. Chrudzimski \& W. Huemer. (Eds.). Phenomenology and Analysis: Essays on Central European Philosophy (pp. 199-214). Frankfurt: Ontos.

Inverso, H. (2014). "El espíritu absoluto debería tener también un cuerpo". Una revisión de la relación entre epoché y corporalidad en Husserl y Merleau-Ponty. Devenires, 29, 63-82.

Inverso, H. (2015). El mundo entre paréntesis. Una arqueología de las nociones de reducción y corporalidad. Buenos Aires: Prometeo. 
Inverso, H. (2016a). Fenomenología de lo inaparente (Tesis doctoral). Universidad de Buenos Aires, Buenos Aires, Argentina.

Inverso, H. (2016b). La fenomenología de lo inaparente y el problema de las vías de acceso a lo trascendental. Eidos, 26, 43-73.

Kern, I. (1977). The Three Ways to the Transcendental Phenomenological Reduction in the Philosophy of Edmund Husserl. En: F. Elliston, et al. (Eds.). Husserl: Exposition and Appraisals (pp. 126-149). Notre Dame: University of Notre Dame Press.

Kuhn, H., Avé-Lallemant, E., \& Gladiator, R. (Eds.). (1976). Die Münchner Phänomenologie. Halle: Phenomenologica.

Luft, S. (2004). Husserl's Theory of the Phenomenological Reduction: Between Life-World and Cartesianism. Research in Phenomenology, 34(1), 198-234.

Mohanty, J. (2003). The Concept of 'Psychologism' in Frege and Husserl. En: D. Jacquette. (Ed.). Philosophy, Psychology and Psychologism (pp. 113-130). Netherlands: Kluwer.

Mohanty, J. (2005). Husserl's Concept of Intentionality. En: R. Bernet, D. Welton, \& G. Zavota. (Eds.). Critical Assessments of Leading Philosophers (vol. 3) (pp. 113-130). London: Routledge.

Nehamas, A. (1985). Meno's Paradox and Socrates as a Teacher. Oxford Studies in Ancient Philosophy, 3, 1-30.

Rabanaque, L. (2011). Actitud natural y actitud fenomenológica. Sapientia, 67, 147-163.

Schuhmann, K. (1997). Philosophy and Art in Munich around the Turn of the Century. Poznan Studies in the Philosophy of the Sciences and the Humanities, 54, 35-51.

Sebestik, J. (2003). Husserl Reader of Bolzano. Husserl's Logical Investigations Reconsidered. Contribution to Phenomenology, 48, 59-81.

Seebohm, T. M. (1991). Psychologism Revisited. En: T. Seebohm, D. Föllesdall, \& J. N. Mohanty. (Eds.). Phenomenology and the formal sciences (pp. 149182). Dordrecht: Kluwer.

Spiegelberg, H. (1982). The Phenomenological Movement. Den Haag: Martinus Nijhoff.

Staiti, A. (2012). The Pedagogic Impulse of Husserl's Ways into Transcendental Phenomenology. Graduate Faculty Philosophy Journal, 33(1), 39-56. 
Walton, R. (2006). La filosofía como ciencia estricta según Husserl. Anuario de fllosofía jurídica y social, 21-53.

Walton, R. (2009). La intencionalidad. Sus modos y desvelamiento. En: N. Corona. (Ed.). La fenomenología. Sus orígenes, desarrollos y situación actual (pp. 11-38). Buenos Aires, Universidad de Buenos Aires.

Xolocotzi, A. (2009). Las cosas de la fenomenología. Notas sobre la idea husserliana de una filosofía científica. Acta fenomenológica latinoamericana, III, 121-137.

Zirión Quijano, A. (1989). La palabra de las cosas. Reflexiones sobre el lema "A las cosas mismas”. En: A. Zirión Quijano. (Comp.). Actualidad de Husserl (pp. 99-123). México: Universidad Nacional Autónoma de México/Fundación Gutman/Alianza Editorial Mexicana.

Zirión Quijano, A. (1990). Breve diccionario analitico de conceptos husserlianos. México: Universidad Nacional Autónoma de México.

Zirión Quijano, A. (2003). La noción de fenomenología y el llamado a las cosas mismas. En: Á. Xolocotzi (Coord.). Hermenéutica y fenomenología. Primer Coloquio (pp. 31-58). México: Universidad Iberoamericana. 